\title{
Noise in (double) relaxation oscillation SQUIDs
}

Derk Jan Adelerhof, Jaap Flokstra, and Horst Rogalla, Department of Applied Physics, University of Twente, P.O. Box 217, 7500 AE Enschede, The Netherlands.

We have modelled the effect of two intrinsic noise sources on the flux noise spectral density of (Double) Relaxation Oscillation SQUIDs ((D)ROSs) based on hysteretic Josephson tunnel junctions. An important noise source is the spread in the critical current of the SQUID due to thermal fluctuations. Critical current noise mainly determines the noise on the average output voltage of DROSs with high flux to voltage transfer. A second noise source is the spread in the relaxation frequency due to the random interaction between the Josephson oscillations and the relaxation oscillations during switching to the zero-voltage state. This effect can dominate the voltage noise of a ROS.

\section{INTRODUCTION}

(Double) Relaxation Oscillation SQUIDs have been demonstrated to be good candidates for SQUID systems with simplified read-out electronics ${ }^{1,2,3}$. In ROSs flux to voltage transfer coefficients up to $4 \mathrm{mV} / \phi_{0}$ have been achieved. In DROSs transfer coefficients of $10-30 \mathrm{mV} / \phi_{0}$ and sometimes even higher have been obtained by the authors ${ }^{2,3}$. These high values enable direct voltage read-out by room temperature electronics.

Two major noise sources will be discussed: critical current noise due to thermal fluctuations and noise caused by the dynamics of the relaxation oscillation mechanism. The effect of these sources on the total intrinsic flux noise of the SQUIDs will be discussed after an introduction to the flux to voltage transfer in ROSs and DROSs.

\section{FLUX TO VOLTAGE TRANSFER}

A ROS consists of a hysteretic de SQUID shunted by an inductance $L$ and a resistance $R$ in series and biased by $a \mathrm{dc}$ bias current ${ }^{1,2}$. The relaxation oscillations can be described by simple analytical equations. From these equations the average voltage as a function of the applied flux can be calculated. For a ROS with $\beta_{\mathrm{L}}=2 \mathrm{I}_{\mathrm{o}} \mathrm{L} / \phi_{\mathrm{o}}=1$, the maximum transfer coefficient from flux to voltage can be calculated to be:

$$
\delta V / \delta \phi_{R O S}=0.9 \frac{2 I_{0} R}{\phi_{0}} .
$$

A DROS consists of a detector SQUID and a reference SQUID in series shunted by an L/R circuit $^{3}$. The average voltage across the reference
SQUID is zero if the critical current $I_{c 2}$ of the reference SQUID is larger than the critical current $I_{c 1}$ of the detector SQUID. At $\mathrm{I}_{\mathrm{c} 2}<\mathrm{I}_{\mathrm{cl}}$ the voltage is nonzero and constant: $\mathrm{V}=\mathrm{V}_{\mathrm{c}}$. The flux to voltage transfer is maximum at $I_{\mathrm{cl}}\left(\phi_{1}\right)=I_{\mathrm{c} 2}\left(\phi_{2}\right)$ and is determined by the spread in the critical currents and the transfer $\delta \mathrm{I}_{\mathrm{cl}} / \delta \phi_{1}$ of the detector SQUID :

$$
\delta V / \delta \phi_{D \text { ROS }}=\frac{V_{c}}{\left(\Delta I_{c 1}+\Delta I_{c 2}\right)}\left(\frac{\delta I_{c 1}}{\delta \phi_{1}}\right)
$$

The spread $\Delta \mathrm{I}_{\mathrm{cl}, 2}$ is due to the finite lifetime of the zero-voltage state caused by thermal fluctuations ${ }^{4}$. An upper limit of $\Delta \mathrm{I}_{\mathrm{cl}, 2}$ is given by the full width at half maximum (fwhm) value of the distribution $P(I)$ of the critical current in a tunnel junction (with $P(I)$ as in fig. 4 of ref. 4) with $I_{c}=2 I_{o}$ calculated for a sweep frequency of the bias current $I_{b}$ which is in agreement with the relaxation frequency in the (D)ROS. In fig. 1 the fwhm values at $4.2 \mathrm{~K}$ of different tunnel junctions at different sweep

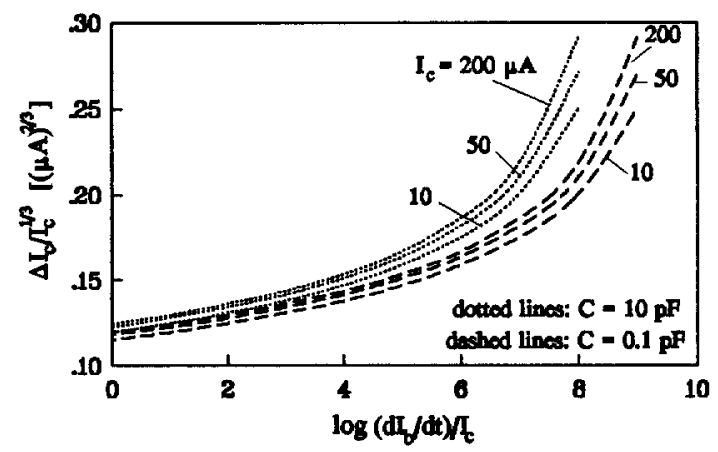

fig. $1 \Delta \mathrm{Ic}$ as a function of the sweep frequency, calculated for junctions with $\mathrm{I}_{c}=20,50$ and $200 \mu \mathrm{A}$ and two different capacitances $\mathrm{C}$. 
frequencies of the bias current are shown. At typical relaxation frequencies of 0.1 to $1 \mathrm{GHz} \Delta \mathrm{I}_{\mathrm{c}}$ is equal to $(0.2-0.3) \mathrm{I}_{c}^{1 / 3}(\mathrm{I}$ in $\mu \mathrm{A})$.

\section{CRITICAL CURRENT NOISE}

In a ROS noise on the critical current is directly transformed to a flux noise $S_{\phi, 1}^{\circ}$ :

$$
\left(S_{\phi}^{I}\right)_{\text {ROS }}=\frac{\sigma\left(I_{c}\right)^{2}}{(\delta I / \delta \phi)^{2}}
$$

where $\sigma\left(I_{\mathcal{C}}\right)$ is the standard deviation of the critical current. Our calculations have shown that $\sigma\left(I_{c}\right)$ is approximately equal to $\Delta \mathrm{I}_{\mathrm{c}} / 3\left(\Delta \mathrm{I}_{\mathrm{c}}\right.$ as in fig. 1). The maximum transfer $\delta I_{c} / \delta \phi$ is about equal to $2.2 \mathrm{I}_{\mathrm{o}} / \phi_{\mathrm{o}}$ in a symmetric de SQUID with $\beta_{\mathrm{L}}=1$.

Due to the critical current noise the output voltage of a DROS biased at $I_{c 1}\left(\phi_{1}\right)=I_{c 2}\left(\phi_{2}\right)$ has equal probability to be oscillating between the gap voltage $V_{g}$ and $0 \mathrm{~V}$ (with average value $V_{c}$ ) or to remain zero during an oscillation cycle. Consequently, the average voltage over a time interval $\Delta t$ much larger than the oscillation period $1 / B$ has a Gaussian distribution with standard deviation $\sigma_{v}=$ $0.5 \mathrm{~V}_{\mathrm{c}} /(\mathrm{B} \Delta \mathrm{t})^{1 / 2}$. This results in a contribution to the flux noise at low frequencies of:

$$
\left(S_{\phi}^{I}\right)_{D R O S}=\frac{V_{c}^{2}}{4 B}\left(8 V / \delta \phi_{D R O S}\right)^{-2} .
$$

\section{RELAXATION OSCILLATION NOISE}

A critical point in the relaxation oscillations of a (D)ROS is the switching from $\mathrm{V}=\mathrm{V}_{\mathrm{g}}$ to $\mathrm{V}=0 \mathrm{~V}$ when the current through the SQUID is close to zero. Because of noise the superconducting phase change across the junctions will have a random value (modulo $2 \pi$ ) every time the SQUID starts to switch back to $0 \mathrm{~V}$. This effect has been simulated numerically for a relaxation oscillation tunnel junction ${ }^{3}$. In fig. 2 two oscillations cycles are shown in which two different phases were chosen at $I=$ $1.6 \mu \mathrm{A}$ and $\mathrm{V} \approx \mathrm{V}_{\mathrm{g}}$. These graphs show that there can be a spread $\Delta t$ in the zero-voltage time $t_{0}$ of each oscillation cycle of about $2 \pi(L C)^{1 / 2}$, where $C$ is the junction capacitance. Assuming two possible values of $t_{0}\left(t_{o 1}\right.$ and $t_{o 2}$, with $\left.\left|t_{o 1}-t_{o 2}\right|=\Delta t\right)$ the maximum

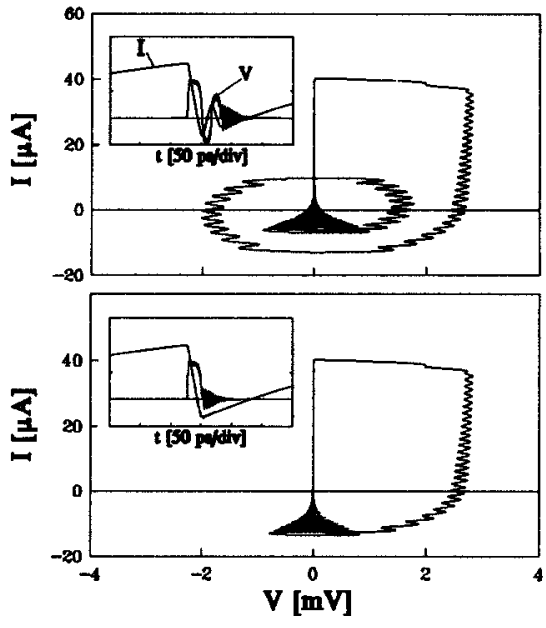

fig. 2 Two different cycles of a relaxation oscillation junction with $\mathrm{C}=0.04 \mathrm{pF}, \mathrm{L}=1 \mathrm{nH}$ and $\mathrm{R}=2.5 \Omega$. The insets show the time dependence of $\mathrm{V}$ and $\mathrm{I}$.

contribution $\left(\mathrm{S}_{\phi}^{\Delta t}\right)_{\max }$ to the flux noise in ROSs and DROSs is equal to:

$$
\left(S_{\phi}^{\Delta y}\right)_{\max }=\frac{\left(V\left(t_{o l}\right)-V\left(t_{o 2}\right)\right)^{2}}{4 B}(\delta V / \delta \phi)^{-2},
$$

where $\mathrm{V}\left(\mathrm{t}_{\mathrm{on}}\right)$ is the average voltage with zero voltage time $\mathrm{t}_{\mathrm{on}}$.

\section{CONCLUSIONS}

We discussed the effect of two intrinsic noise sources on the flux noise of ROSs and DROSs with voltage read-out. In ROSs the random interaction between the Josephson frequency and the relaxation dynamics can dominate the output voltage noise if the ratio of the relaxation oscillation period $1 / B$ and $(\mathrm{LC})^{1 / 2}$ is too small. In DROSs with high flux to frequency transfer, the effective flux noise is dominated by the noise on the critical current.

\section{REFERENCES}

1) S.A. Gudoshnikov et al, IEEE Trans Magn. 27 (1991) 2439.

2) D.J. Adelerhof et al., accepted. for publ. in IEEE Trans Appl. Supercond. 27 (1993).

3) D.J. Adelerhof et al., to be published.

4) T.A. Fulton et al., Phys Rev. B 9 (1974) 4760. 